\title{
L'imprenditore innovatore come agente dello sviluppo tecnico, economico e sociale ${ }^{*}$
}

\author{
ALFONSO GAMBARDELLA
}

\begin{abstract}
Obiettivo del paper: L'obiettivo della seguente relazione è di analizzare la figura dell'imprenditore innovatore descrivendo le principali caratteristiche che lo rendono uno tra i più importanti agenti dello sviluppo tecnico, economico e sociale di un Paese.

Metodologia e risultati: Quella che segue è un'analisi qualitativa che fa emergere il razionale teorico del perché la figura dell'imprenditore sia fondamentale all'interno di un ecosistema dell'innovazione e che porta alla luce alcuni tra gli esempi più eclatanti sul modo in cui tale figura si è fatta colonna portante del cambiamento e dello sviluppo economico e sociale nel mondo.

Limiti della ricerca: Un più ampio resoconto delle analisi empiriche condotte in questi anni per misurare $i$ benefici generati dall'imprenditoria potrebbe arricchire la discussione avviata in queste pagine.

Implicazioni pratiche e Originalità del lavoro: Anche la semplice panoramica offerta in questo documento ha il potenziale di fare da eco alla necessità che ogni Paese ha di coltivare il talento e incoraggiare una maggiore attività imprenditoriale. In particolare, il seguente paper incarna il tentativo di fornire un'utile mappa concettuale nel quale analizzare $i$ diversi canali tramite cui l'imprenditore influenza ed è influenzato dal sistema tecnico, economico e sociale che lo circonda, fungendo da spunto per future analisi empiriche volte a studiare la relativa importanza di ognuno di questi canali.
\end{abstract}

Parole chiave: imprenditore; innovazione; sviluppo

Purpose of the paper: The aim of the following report is to analyze the figure of the entrepreneur-innovator describing the main features that make him one of the most important agents of technical, economic and social development in a country.

Methodology and Results: The following is a qualitative analysis that either reveals the theoretical rationale for why the entrepreneur represents such a crucial factor within an ecosystem of innovation and brings to light some of the most striking examples of the way in which the entrepreneur has become one of the main triggers of economic and social development in the world.

* Un ringraziamento particolare ad Alessandro Cordova per i commenti e la discussione che hanno portato alla stesura di questa relazione. Resto naturalmente il solo responsabile dei contenuti.

** $\quad$ Ordinario di Economia e Gestione delle Imprese - Università Bocconi e-mail: alfonso.gambardella@ unibocconi.it

sinergie, rivista di studi e ricerche

n. 93 , Gennaio-Aprile 2014, p. 3-18

ISSN 0393-5108- DOI 10.7433/s93.2014.02 
Limitations of the research: A comprehensive account of the empirical studies conducted in order to state quantitatively the benefits generated by entrepreneurs might enrich the discussion initiated in these pages.

Practical implications and originality of the work: Even the simple overview offered in this document has the potential to echo the need that each country has to nurture talent and encourage entrepreneurship. In particular, the following paper embodies an attempt to provide a useful conceptual map in which to analyze the various channels through which the entrepreneur influences and is influenced by the technical, economic and social environment he/she is surrounded by, serving as a starting point for future empirical analysis aiming at studying the relative importance of each of these channels.

Key words: entrepreneur; innovation; development

\section{L'Innovazione e l'Imprenditore}

L'innovazione è uno dei fattori che contribuisce maggiormente alla crescita economica, culturale e sociale di ogni Paese. Nuovi processi produttivi, nuove tecnologie, nuovi modelli organizzativi, permettono di accrescere la produttività delle imprese ${ }^{1}$, di generare efficienza nel sistema ${ }^{2}$, di ottimizzare l'allocazione delle risorse disponibili ${ }^{3}$, di adattare conoscenze e risorse esistenti in diversi settori dell'economia, generando una crescita inclusiva e sostenibile ${ }^{4}$.

La domanda è dunque la seguente: Come si genera innovazione? Quali sono i mezzi attraverso cui diffonderla e permettere quindi lo sviluppo tecnico, economico e sociale di un Paese?

La risposta a queste domande può essere lunga e complessa. Tuttavia, volendo deliberatemene semplificare il quadro per offrire un primo messaggio semplice e chiaro, Schumpeter sosteneva con forza che il veicolo dell'innovazione fosse l'imprenditore.

1 Si pensi che l'innovazione ha contribuito per il $63 \%$ della crescita in produttività del lavoro generata nel Regno Unito negli anni 2000-2008, NESTA (2012).

2 Ad esempio, l'impiego delle tecnologie ICT all'interno della PA può comportare la riduzione dei tempi e i costi che la burocrazia comporta per i cittadini e le imprese.

3 L'innovazione che diminuisce $\mathrm{i}$ tempi di lavoro ma mantenendo eguale o migliorando la qualità e il volume della produzione permette di liberare spazi che l'uomo può dedicare ad altre attività. L'aumento di produttività nel settore manifatturiero, ad esempio, ha portato all'impiego di una parte crescente della forza lavoro all'economia dei servizi.

4 Un esempio di re-impiego di tecnologie esistente in altri campi da quello originale di applicazione, è quello di Marks \& Spencer. L'azienda multinazionale inglese aveva constatato che una parte molto importante nella preparazione dei sandwich era la spalmatura del burro sul pane, un'attività alquanto labor intensive. Fu così che un responsabile notò come un processo serigrafico usato da un fornitore per la stampa di motivi su biancheria da letto, potesse essere adattabile alla spalmatura del burro sul pane, comportando quindi un importante taglio dei costi all'interno del business e quindi una crescita dell'azienda (Aaker, 2011). 
L'imprenditore riconosce l'innovazione nel suo stato primordiale, ne vede e realizza il potenziale, guidato dal motivo economico e dal desiderio di mettere a frutto le proprie capacità. Introduce nuovi prodotti, sfrutta le innovazioni tecnologiche, apre nuovi mercati e, così facendo, genera sviluppo economico. Assume inoltre un ruolo fondamentale all'interno del ciclo economico (Schumpeter, 1911). Tutto ha inizio da un modello in cui il punto d'origine è lo "stato stazionario", la fase in cui le imprese realizzano soltanto attività routinarie e il sistema economico replica se stesso nel tempo, senza che ci sia creazione di vera ricchezza. Lo sviluppo economico inizia quando un imprenditore stravolge lo stato stazionario attraverso l'introduzione di un'innovazione, ovvero una nuova combinazione di vecchie e nuove conoscenze. L'imprenditore è dunque la variabile che sciocca il sistema economico e mette in moto l'innovazione.

Ma come si tramuta l'attività innovatrice dell'imprenditore in un impatto economico e sociale sulla comunità?

Se più avanti parleremo dell'influenza per così dire "diretta" che l'attività imprenditoriale impartisce e, allo stesso tempo, subisce dalla comunità che lo circonda, portando alla luce episodi di investimenti che le imprese stanno conducendo nel sociale e citando esempi di imprenditori che si sono fortemente identificati con la comunità, illustriamo ora quello che è l'impatto "indiretto": l'innovazione come "distruzione creatrice" (Schumpeter, 1942), un processo in cui la novità comporta la scomparsa dell'obsoleto, un fenomeno che da un lato induce la nascita di nuove imprese e di nuovi mercati, incidendo sull'assetto industriale di interi settori, e dall'altro modifica i prodotti e i prezzi offerti ai consumatori, incidendo sul loro benessere.

\section{L'Innovazione come distruzione creatrice}

Schumpeter (1941) fa un esempio per illustrare il fenomeno dell'innovazione come distruzione creatrice: la costruzione delle ferrovie nel Middle West intrapresa dall'Illinois Central. Traducendo il correspettivo passo contenuto nel suo scritto, si legge come questo investimento, che rappresentava l'introduzione di una nuova innovazione (il trasporto su ferrovia) a scapito del vecchio sistema della autocorriera, significasse sì una straordinaria opportunità di sviluppo economico di nuove aree, poiché nuove città venivano edificate e la terra coltivata, ma allo stesso tempo la condanna a morte della (vecchia) agricoltura dell'Occidente.

Questo fenomeno di distruzione creatrice è in continua riproduzione.

Un episodio recente è quello della cassetta audio, le cui minor dimensioni e capacità di riavvolgimento del nastro ne determinarono il successo rispetto al vecchio 8-track format, successivamente sostituita dai compact disk e dai lettori MP3. Anche Internet ha agito come catalizzatore per la distruzione creativa: i giornali e i giochi online, ad esempio, hanno cominciato a sbaragliare la concorrenza dell'editoria classica e dei giochi su cd-rom. Così anche il mondo delle app sugli smartphone sta rivoluzionando molti settori: un esempio è whatsapp che, come i 
social network Facebook e Skype, ha ridotto il costo di comunicare (messaggistica gratis), spingendo molte persone a preferire tale servizio alla messaggistica a pagamento del telefono.

Il processo di distruzione creatrice non è tuttavia innescato unicamente da innovazioni così dette radicali, ovvero che rivoluzionano completamente sia le componenti che l'architettura di un prodotto, come nel caso del CD rispetto alla cassetta.

Anche forme di innovazione così dette "architetturali" (Clark e Henderson, 1990), ovvero che modificano la struttura ma non i singoli componenti di un dato prodotto, possono dare vita a frequenti e importanti cambiamenti in un determinato mercato. Queste innovazioni rappresentano un cambiamento sostanziale nel modo di integrare tra loro tecnologie e le componenti-chiave dei prodotti (Torrisi, 2011). Consideriamo un primo esempio fatto da Clark ed Henderson: il ventilatore da soffitto. Mentre un'innovazione radicale in questo mercato potrebbe essere rappresentata dalla commercializzazione dei condizionatori, un esempio di innovazione architetturale è quella dell'introduzione del ventilatore "da tavolo". In questo caso, mentre le componenti del ventilatore (pala, motore, ecc.) rimangono le stesse sia nel ventilatore da soffitto che da tavolo (a differenza del condizionatore dove le pale scompaiono), l'architettura del prodotto cambia: nel ventilatore da tavolo, la co-localizzazione del motore e delle pale all'interno dello stesso corpo e le minori dimensioni dello stesso, trasformano le interazioni tra il motore, le pale e quindi la quantità d'aria che le pale possono far circolare, ecc.

Questo tipo di innovazione, allo stesso modo di quella radicale, comporta delle sfide non indifferenti per le imprese esistente sul mercato: poiché le "vecchie" imprese maturano un'estesa esperienza e conoscenza nel "vecchio prodotto", esse non sempre riescono ad adottare nuove innovazioni architetturali introdotte dalle nuove aziende. Clark ed Henderson fanno due esempi di questa "rigidità" o "inerzia" tipica delle imprese consolidate rispetto ai nuovi entrants nel mercato.

Il primo è quello dell'aereo a reazione, dove le imprese operanti sul mercato dell'aviazione compresero presto l'importanza di sviluppare esperienza nel nuovo settore del motore a getto, ma fallirono nel comprendere a fondo che tipo di nuove "interazioni" questa nuova tipologia di motore implicasse con le restanti componenti dell'aereo (Miller and Sawyers, 1968; Gardiner, 1986), ovvero come sarebbe cambiato il rapporto carburante-velocità del velivolo, piuttosto che il rapporto grandezza del velivolo-potenza del motore. Questo "fallimento" fu una delle ragioni per cui la neo-fondata Boeing riuscì ad attestarsi alla leadership dell'industria.

Ancora più esteso è l'esempio che gli autori fanno dell'industria della litografia elettronica (o fotolitografia). Tecnologia chiave per le industrie di semiconduttori, la fotolitografia è quel processo che vede il trasferimento di un pattern da una maschera ad un sottile strato di materiale sensibile alla radiazione (chiamato resist) che copre la superficie di un substrato, come ad esempio una fetta di semiconduttore. Mentre i primi allineatori mettevano a contatto la maschera con il semiconduttore, quelli successivi (detti 'di prossimità') evitavano il contatto, migliorando la qualità del processo litofotografico (Torrisi, 2011). L'azienda leader 
negli allineatori a contatto (introdotti per la prima volta nel 1973), Kasper Instrument, non riuscì a passare rapidamente alla produzione di allineatori di 'prossimità' (ideati dalla neo-fondata Canon alla fine degli anni ' 70 con minime modifiche ai vecchi allineatori), perché i suoi ingegneri li considerarono una minima innovazione incrementale, una "evoluzione routinaria" nella linea di prodotto degli allineatori a contatto ${ }^{5}$. Allo stesso modo, le prime lamentale da parte dei clienti riguardo alla limitata precisione e stabilità del meccanismo di gap-setting degli allineatori a contatto, ovvero il meccanismo di separazione tra la maschera e il semiconduttore durante l'allineamento (l'elemento che più differenzia le due versioni di allineatori), fu creduto essere causato da un semplice "processing error". Di conseguenza, in risposta all'innovazione introdotta da Canon, Kasper modificò solo parzialmente il meccanismo di gap-setting, senza mai comprendere a fondo l'importanza che la sua accuratezza (la maschera deve essere posizionata in un punto preciso sopra al semiconduttore) comportava per la performance dell'allineatore. Ciò contribuì al rapido declino nelle vendite di Kasper e alla sua fuoriuscita dal mercato agli inizia degli anni ' 80.

Un altro tipo di innovazione che può rivoluzionare un intero settore è quello delle innovazioni così definite trajectory-disrupting, ovvero tecnologie che, invece di generare il miglioramento di prodotto atteso dai consumatori nei mercati di punta, aprono nuovi segmenti di mercato; quest'ultimi vengono inizialmente considerati "inferiori" dalle aziende che operano nei mercati di punta perché analizzati attraverso gli standard tradizionali, ma in realtà nascondono un forte potenziale innovativo.

Questo è il caso dell'industria dei disk drive (Christensen, 1993), dove quelli a 14 pollici sono stati sostituiti prima da quelli a 8 pollici, poi da quelli a 5,25 e da 3,5. I produttori di disk drive a 14 pollici, non furono in grado di captare l'importanza dei nuovi disk drive prodotti da nuovi entrants nel mercato perché i propri clienti, i produttori di mainframe, avevano un fabbisogno diverso da quello dei clienti delle nuove imprese. Quest'ultime, infatti, vendevano a produttori di minicomputer, allora una semplice nicchia di mercato. I minicomputer non avevano "bisogno" di disk drives ad alta velocità e ampia capacità al pari dei mainframes, ma piuttosto puntavano a disk drives di inferiori dimensioni e che fossero più economici. Quando il mercato dei minicomputer iniziò a crescere rapidamente, i vecchi leader del settore dei disk drive furono pian piano sostituiti dai nuovi entrants. In altre parole, le imprese di successo nella produzione dei disk drive a 14 pollici si erano focalizzati unicamente sulla loro rete di clienti attuale, puntando sul miglioramento delle proprietà del proprio prodotto piuttosto che sulle tecnologie emergenti.

Se l'innovazione descritta sino ad ora è apparsa sotto forma di un nuovo prodotto/mercato, bisogna riconoscere che vi sono diverse forme di innovazione che influiscono sulla comunità: innovazione vuol dire anche introduzione di nuovi processi produttivi, trasformazione dei settaggi organizzativi/manageriali

5 Ancora una volta colpa del fatto che la nuova tecnologia fu interpretata sulla base di vecchi criteri, ovvero quelli impiegati per valutare i vecchi allineatori. 
dell'azienda, nonché modelli di business ${ }^{6}$. Anche in questo caso, l'innovazione opera come "distruzione creativa" che incide sulla comunità.

\section{L'Innovazione come nuovi modelli di business}

Introdurre un business model ${ }^{7}$ innovativo può essere determinante per il successo di un'impresa e può determinare il riassetto di un intero settore.

Starbucks ne è un esempio.

Nata nel 1971, la compagnia statunitense ha rivoluzionato il business del caffè ed il suo brand è diventato in poco tempo uno dei più riconosciuti a livello internazionale di sempre.

Ispirata dalla visione italiana del caffè come un'esperienza di condivisione, Starbucks ha raccolto l'eredità del baby-boom americano degli anni '40-'60 e ha offerto, al crescente numero di giovani professionisti negli Stati Uniti e per gli studenti delle grandi metropoli, un luogo dove vivere una "affordable luxury": un'esperienza di comfort, di relax e di condivisione. Ed è proprio lo store il primo segnale del business model innovativo di Starbucks: se la vecchia credenza era che intrattenere il cliente in negozio implicasse l'affollamento dello stesso e quindi una riduzione nel volume delle vendite, l'intrattenimento diventò il principale veicolo di costruzione e rafforzamento del brand di quest'azienda.

L'impatto che Starbucks ha generato per la comunità non si limita a quello sui consumatori.

In primis, Starbucks ha riconosciuto un grande valore ai propri dipendenti, comprendendo l'importanza di mettere a disposizione incentivi per aumentare la loro produttività. A seconda delle ore di lavoro annue e il periodo di lavoro totale trascorso presso l'azienda, i dipendenti ottengono: un'estesa copertura assicurativa sulla salute; la possibilità di contribuire una parte compresa tra l'1 e il $75 \%$ del proprio stipendio (con un tetto massimo attorno ai 15-20 mila euro l'anno) all'interno del fondo pensione di Starbucks, che si impegna a contribuire una somma eguale a quella versata dal dipendente; sconti sull'acquisizione delle azioni della compagnia, nonché un premio in azioni annuale sulla base della performance dell'azienda; benefici per l'istruzione, come la possibilità di guadagnare crediti scolastici al college a patto di aver completato il training da Starbucks, sconti e/o rimborsi sui libri di testo e persino sui corsi universitari; una serie di altri benefici

6 Lo stesso Schumpeter (1911) riconosce 5 tipi di innovazione: introduzione di un nuovo prodotto o servizio, nuovo processo produttivo, aperture di nuovi mercati, l'accaparramento di una nuova fonte di materie prime, il cambiamento nella struttura di un industria.

7 Definito come il riflesso della strategia dell'impresa, quindi l'espressione della logica dell'azienda, del suo funzionamento e del suo modo di creare valore per gli stakeholder (Baden-Fuller et al., 2010). 
come sconti per prodotti o servizi acquistati da imprese locali e programmi di wellness.

Secondo, Starbucks è stata una delle prime imprese a lanciare una campagna di fair trade per il caffè, dando un forte impulso alla sua immagine di azienda impegnata nel sociale. La prassi del fair trade prevede l'acquisizione del caffè direttamente da gruppi di piccoli agricoltori del Terzo Mondo, invece che dai grandi intermediari, con il rispetto delle regole del "commercio equo", che assicurano ai piccoli produttori un prezzo minimo di vendita che possa garantire loro la sopravvivenza. Oltre a questo impegno, ve ne sono altri nel campo della preservazione dell'ambiente, come l'utilizzo crescente di carta riciclabile, gli sconti ai clienti che portano da casa la propria tazza per bere il caffè, l'offerta gratis di fondi di caffè alle famiglie che hanno bisogno di fertilizzante per i propri giardini, ecc.

Nuovi business models sono nati anche in settori come la finanza, vedi la Grameen bank in Bangladesh.

La Grameen bank, nata in Bangladesh nel 1983 dal Professore Muhammad Yunus, vincitore del Nobel per la pace insieme alla sua organizzazione nel 2006, è diventata uno dei primi esempi di impresa sociale: una formula di business che coniuga il profitto con l'utilità sociale.

L'idea di fondare la Grameen bank nacque, come spesso accade, da un episodio. Parlando con una donna del villaggio di Jobra, Sufiya Begum, egli scoprì che, come molte altre persone, Sufiya era costretta a prendere in prestito il denaro dagli strozzini del villaggio per mandare avanti il proprio business (lavorare il bambù per la produzione di sgabelli), vista che le banche non concedevano prestiti in mancanza di collaterale ${ }^{8}$. Gli strozzini le imponevano condizioni inaccettabili: in cambio della concessione del prestito, chiedevano che Sufiya vendesse loro tutto ciò che avrebbe prodotto e a prezzi miserrimi. Così come Sufiya, tantissimi imprenditori dei villaggi del Bangladesh si trovavano costretti in una vera e propria "trappola della povertà". Fu allora che Yunus decise di prestare lui stesso il denaro a Sufiya. Con sua grande sorpresa, lo riottenne in una sola settimana. Fu cosi che cominciò a prestare piccole somme di denaro ad altri imprenditori in difficoltà, rendendosi conto che l'imprenditoria era una qualità assolutamente non da meno presente nelle classi meno abbienti (ad oggi il tasso di rimborso è attorno al 98\%). Nonostante altre situazioni di questo tipo si ripeterono, le banche rimasero riluttanti a prestare denaro e per questo motivo Yunus decise di dare vita a quello che oggi rappresenta uno degli strumenti di microcredito più importante al mondo. Il business model della Grameen bank re-inventò le regole del gioco: sfidò l'assunzione che i tassi di rimborso dei prestiti concessi alle classi meno abbienti fossero decisamente più bassi di quelli ad imprenditori affermati con molto capitale a disposizione. Inoltre, Yunus

8 Oltre alla mancanza di collaterale, la maggior parte degli abitanti di questi villaggi non avevano alcuna storia creditizia e, anche se ne avessero avuta una e avessero disposto del collaterale richiesto, non avrebbero potuto firmare i documenti necessari per ottenere il prestito poiché analfabeti. 
notò che gli imprenditori meno abbienti erano dotati di una grande qualità: si aiutavano a vicenda. Fu così che fu introdotta una formula di prestito che facesse forza del principio di peer pressure: i prestiti venivano concessi a gruppi di imprenditori e solo se $\mathrm{i}$ primi due soggetti in un gruppo iniziavano a rimborsare il capitale e gli interessi entro sei settimane dall'elargimento del credito, allora gli altri membri del gruppo potevano anch'essi ottenere dei prestiti (Yunus et al., 2010).

Nuovi business models non si affermano solo all'interno di singole imprese ma anche a livello di settore.

Questo è il caso del crescente utilizzo, negli ultimi anni nei settori high-tech, della concessione in licenza di general-purpose technologies (Gambardella e McGahan 2010). Mentre negli anni '70/'80 le aziende davano in licenza la propria proprietà intellettuale prevalentemente ad imprese cross-border, come strumento per ottenere rents da mercati in cui non si disponeva di una presenza diretta, negli anni '90 lo scambio di tecnologie cominciò ad espandersi anche all'interno delle stesse aree geografiche. Il fenomeno ha iniziato a prendere piede negli Stati Uniti, quando start-up nell'industria dei software iniziarono a dare in licenza le loro tecnologie a grandi imprese operanti nello stesso settore; la ragione alla base di questo fenomeno era riconducibile al fatto che queste grandi imprese disponevano di una maggiore esperienza nella distribuzione e commercializzazione del prodotto finale (interface design, distribuzione al dettaglio, assistenza post-vendita, attività di marketing, ecc.). Man mano che questa attività si mostrò profittevole, molte imprese di software entrarono nel mercato con l'espressa intenzione di vendere/dare in licenza le loro applicazioni. Successe la stessa cosa nell'industria delle biotecnologie. Negli anni '80, l'obiettivo di ogni impresa in questo settore era quello di diventare un'affermata casa farmaceutica, ma molte imprese erano ostacolate nella propria capacità a valle (le stesse abilità di commercializzazione del prodotto finale descritte per il settore dei software). Per questo motivo, molte di esse cominciarono a vendere i propri composti farmacologici a grandi imprese farmaceutiche. Tuttavia, questi composti avevano applicazioni specifiche, il che limitava il profitto che queste piccole imprese biotecnologiche potevano ottenere: i guadagni potenziali dipendevano dalla capacità della grande imprese di avere successo sul mercato; inoltre, sempre per via della limitata applicabilità del composto, le piccole aziende biotech avevano un limitato potere contrattuale nei confronti delle grandi imprese. Fu cosi che negli anni '90, aziende come Affymetrix ed ECI Biotech, smisero di vendere composti specifici ed iniziarono a dare in licenza formule più generali, ovvero strumenti di ricerca come dispositivi di bioinformatica, le tecnologie di screening, simulatori per testare i farmaci, ovvero strumenti utili per un'ampia gamma di applicazioni farmaceutiche. Questo fenomeno ha il potenziale di avere un impatto significativo sulla comunità (indiretto) in quanto la crescente disponibilità sul mercato di conoscenze e tecnologie costringe ad una maggiore competizione nell'elaborazione e accaparramento di nuove idee, dando un forte impulso all'innovazione a catena (tradotto per la comunità può significare progresso economico, tecnico e sociale). 
L'innovazione intesa come nuovi business models non è unicamente prodotta nei settori a maggior tasso tecnologico. Un ulteriore esempio, oltre quello della Grameen bank, è quello delle due catene di abbigliamento Zara ed H\&M.

Le due società di distribuzione tessile avevano visto che esisteva una grande domanda "insoddisfatta" per capi d'abbigliamento all'ultima moda da parte di giovani vista la loro limitata capacità d'acquisto rispetto ai prezzi imposti delle grandi marche. Per questo motivo deciso di risolvere il problema dando vita ad una formula chiamata "fast fashion": sono capi che si rifanno alle ultime tendenze espresse dal mondo del fashion o all'indomani delle sfilate stagionali, ma ad offerti ad un moderato prezzo di vendita. Inoltre lavorano in modo differente dai negozi di abbigliamento che vendono capi realizzati in Cina e in altri paesi che forniscono manodopera a basso costo. Il design e la produzione di Zara, per esempio, avvengono in Spagna o nel nord del Portogallo, in aree dove i salari sono bassi. In questo modo prendono vantaggio del più elevato tasso di skills di questi lavoratori e al contempo offrono opportunità di lavoro in aree a minor crescita economica. Un'altra formula vincente di questi brand è la velocità nel cambiamento dell'offerta. In altre parole, i capi venduti nei punti vendita cambiano continuamente (la maggior parte dei capi sono disponibili per un mese). Questo fa si che i clienti siano incentivati ad andare spesso in negozio per monitorare le novità, una strategia di marketing che rende la sola presenza del punto vendita sufficiente a sostenere il brand, senza bisogno di pubblicità (Aaker, 2011). Anche in questo, un nuovo business models ha creato vantaggi per i consumatori, per l'impresa e anche per la comunità, vista la produzione dei capi d'abbigliamento in Spagna e Portogallo.

L'innovazione si presenta infine anche sotto forma di vecchi modelli di business re-inventati.

Rossopomodoro e Grom, nel settore alimentare, rientrano in questa categoria. Nel mondo della globalizzazione, delle multinazionali e delle produzioni di massa, gli imprenditori delle due catene alimentari, hanno maturato la consapevolezza del desiderio dei consumatori di tornare a comprare prodotti fatti alla vecchia maniera, lavorati a mano e di primissima qualità?

Rossopomodoro utilizza solo mozzarella d.o.p., olio d'extravergine d.o.p. da olive di Sorrento e farina tradizionale di Napoli Caputo, prodotti vincolanti nei contratti di Franchising. Grom usa le nocciole che vengono da Langhe, le pesche di Leonforte, i pistacchi di Bronte e per fare i sorbetti viene impiegata unicamente l'acqua minerale San Bernardo. Allo stesso modo, le materie prime utilizzate, oltre a quelle ricercate sul territorio, sono coltivate con metodo biologico nei terreni di proprietà dell'azienda situati a Castigliole d'Asti (At). A testimonianza dell'importanza data alla qualità da queste due attività imprenditoriali, Rossopomodoro ha istituito una company university, un centro interno di formazione per qualificare lo staff allo standard da mantenere nei propri locali.

9 Per quanto riguarda Grom, per esempio, la realizzazione del gelato comporta un processo quotidiano di lavorazione che possiamo definire artigianale e che non utilizza additivi quali emulsionanti, coloranti, conservanti e aromi sia naturali sia artificiale. 


\section{L'imprenditore e la comunità: l'influenza diretta}

Dopo avere affrontato il tema dell'innovazione come distruzione creatrice, sia sotto forma di nuovi prodotti e processi produttivi che di nuovi modelli di business, e dunque dell'influenza indiretta dell'innovazione sulla comunità, giungiamo ora a quella che potremmo chiamare l'influenza diretta dell'imprenditore sullo sviluppo economico e sociale.

Il crescente intento delle imprese di impegnarsi a favore dell'innovazione sociale è motivato sia dalla volontà dell'imprenditore di contribuire allo sviluppo sociale della comunità, che dalla possibilità di guadagnarne un beneficio, una situazione che gli economisti coniano come win-win: impegnandosi per il bene della comunità, l'imprenditore ha maggiori probabilità di sopravvivere nel tempo, grazie alla distinzione del proprio brand. Allo stesso tempo, la comunità beneficia dagli investimenti compiuti dalle imprese sul territorio ed è quindi maggiormente disposta ad "affiliarsi" a quell'impresa.

È per questo motivo che gli imprenditori stanno agendo sempre più in simbiosi con la comunità, promuovendo attività di corporate giving, strategic philanthropy, social sponsoring ${ }^{10}$, corporate citizenship e anche attività di beneficienza ${ }^{11}$ (Fosfuri et al., 2010).

Uno degli esempi più rappresentativi è quello di Hawlett Packard nell'ambito del supporto all'istruzione.

HP ha creato un ufficio dedicato al "Global Social Innovation", ovvero allo sviluppo e innovazione sociale nel mondo. In oltre 60 anni, HP ha investito milioni di dollari per mettere a disposizione le proprie innovazioni in iniziative educative, ambientali e sanitarie in decine di paesi in tutto il mondo. Nel campo dell'educazione, in particolare, HP ha formato un network (il cosi detto "Catalyst Initiative") che unisce i maggiori esponenti di HP con le scuole e i governi locali di tutto il mondo - in particolare dei paesi più poveri - con l'obiettivo di introdurre innovazioni tecnologiche nel percorso educativo di materie come scienze, tecnologia, ingegneria e matematica (così detti STEM). Si tratta, ad esempio, di dare la della possibilità agli studenti in scuole che non hanno fondi a sufficienza per attrezzarsi di laboratori di ricerca propri, di condurre esperimenti utilizzando

10 Anche le banche sono attivissime nel campo del corporate responsibility, e.g. BNP Paribas é sponsor del Roland Garros, Barclays della Premiership, etc.

11 Alcuni esempi: la Bill Gates Foundation, Chiquita e la sua campagna "L'ambiente giusto fa sviluppo" e il supporto alla COOPI-Cooperazione Internazionale, la campagna "Riscriviamo il Futuro", realizzata dalla Fila, 3M Scotch-Brite sostiene l'UNICEF in un progetto a favore delle bambine del Niger, la politica di fair trade coffee lanciata da Starbucks, in Italia Della Valle destina 1'1\% degli utili a onlus che operano sul territorio fiorentino, etc. In aggiunta, lo stesso fatto che sia nata nell'ordinamento giuridico italiano, la figura dell'impresa sociale, “... attività economica organizzata al fine della produzione e dello scambio di beni o servizi di utilità sociale, diretta a realizzare finalità di interesse generale" secondo l'art.1 del d.lgs.155/2006, è un segnale del crescente interesse degli imprenditori per lo sviluppo sociale della comunità. 
remotamente (online) i laboratori scientifici di università che mettono a disposizione le proprie tecnologie, come la Northwestern University ${ }^{12}$; di dare la possibilità agli studenti in diverse parti del pianeta, di collaborare a progetti scientifici per trovare soluzioni a grandi problemi come lo smaltimento dei rifiuti e l'inquinamento. Per esempio, nel 2010 gli studenti della scuola media Scofield Magnet, di Stanford in Conneticut (US), hanno collaborato con gli studenti della scuola media cinese Shandong University, per risolvere il problema della contaminazione delle acque dei pozzi del proprio paese. Questo è stato reso possibile da HP grazie alla creazione di un portale online per la comunicazione tra le due scuole e la messa a disposizione delle tecnologie necessarie per monitorare i dati sulla qualità delle acque, la topografia, il drenaggio, la flora e la fauna ${ }^{13}$. E così ci sono tanti altri esempi di interventi che HP ha intrapreso nel campo dell'istruzione, come un programma di studio online per studenti aspiranti imprenditori (HP LIFE e-Learning program), per aiutarli ad ottenere le skills necessarie (in campi che vanno dalla finanza al marketing, da operations a comunicazione e IT) per lanciare i propri progetti con successo $^{14}$.

Se le imprese stanno ponendo una crescente attenzione sul tema del corporate and social responsibility, è ancora più significativa l'esperienza di singoli imprenditori che hanno profondamente influito nello sviluppo economico e sociale della propria comunità.

Un esempio su tutti è stato l'imprenditore italiano Adriano Olivetti.

Adriano Olivetti è stata una figura poliedrica - industriale, intellettuale, urbanista, editore - un imprenditore capace di innovare non solo in ambito industriale ma anche in ambito organizzativo e sociale.

Tra le sue convinzioni vi era quella che il fine dell'impresa non dovesse essere limitato al profitto ma al bene della comunità. Olivetti si soffermò molto sull'aspetto umanistico: per essere produttivo, il lavoratore doveva essere coinvolto nei progetti dell'azienda, doveva arrivare ad indentificarsi con l'impresa e i suoi obiettivi, e dunque doveva sentirsi apprezzato e valorizzato da essa. Per questo motivo, le fabbriche, in particolare quella storica di Ivrea, furono dotate di biblioteche, mense, ambulatori medici e asili nido. L'intenzione era quella di dare agli operai l'opportunità di studiare e di usufruire di una serie di servizi funzionali al loro benessere.

I dipendenti Olivetti inoltre avevano salari superiori del 20\% della base contrattuale, alle donne venivano concessi nove mesi di maternità retribuita (un po' meno del doppio del valore odierno), il sabato venne lasciato libero, prima ancora di ogni contrattazione sindacale e, infine, l'orario di lavoro venne ridotto da 48 a 45 ore settimanali, a parità di salario, in anticipo sui contratti nazionali di lavoro.

12 Informazioni estratte dal primo sito web citato nella bibliografia.

13 Informazioni estratte dal secondo sito web citato nella bibliografia.

14 Informazioni estratte dal terzo sito web citato nella bibliografia. 
Anche la politica di assunzione del personale era del tutto peculiare; Adriano in persona seleziona i candidati valutando, oltre ai loro curricula, elementi quali la grafia o il portamento.

Ai giovani veniva riconosciuta un'importanza primaria, perché entusiasti e lontani da una mentalità consueta.

Anche in ambito prettamente economico gestionale, Olivetti avviò una grande opera di modernizzazione: l'organizzazione decentrata del personale, la direzione per funzioni, la razionalizzazione dei tempi e metodi di montaggio, lo sviluppo della rete commerciale in Italia e all'estero, l'importanza data all'aspetto commerciale, alla ricerca e persino al design.

In poco più di un decennio la formula Olivetti portò ad un incremento della produttività del $500 \%$ e un aumento nel volume delle vendite del $1300 \%$. In particolare nel campo dell'elettronica, Olivetti rappresenta tutt'oggi uno dei rari casi in cui l'Italia è stata all'avanguardia nell'innovazione tecnologica e scientifica ${ }^{15}$.

Se Olivetti ha scommesso fortemente sullo "sviluppo" e il benessere della forza lavoro, Aristide Merloni, uno dei maggiori esponenti nel settore elettrodomestico e termoidraulico italiano e mondiale, si è impegnato a fondo per sostenere lo sviluppo economico e sociale del territorio. La sua più grande vocazione fu quella di creare un forte tessuto imprenditoriale in una regione che non ne era dotata: la sua regione di origine, le Marche. Luoghi fino allora remoti come Fabriano, Albacina, Matelica, Borgo Tufico, diventarono sede dei suoi stabilimenti, tanti mono-prodotto disseminati sul territorio; nacque la famosa "via marchigiana allo sviluppo", un modello d'impresa studiato in tutto il mondo come esempio di integrazione armoniosa tra industria e territorio ${ }^{16}$.

L'imprenditore è dunque una figura fortemente carismatica che, motivata dal desiderio di eccellere e di cambiare le regole del gioco, mette a frutto la propria energia ed ingegno per opporsi a quella che Schumpeter definisce inerzia sociale, ovvero le resistenze psicologiche e sociali che si oppongono al cambiamento.

Ma se è vero però che l'imprenditore plasma la realtà economica e sociale che lo circonda, è anche vero il contrario.

\section{La comunità come variabile che incide sulla figura dell'imprenditore}

In un momento storico di forte crisi economica, sia la figura dell'imprenditore che quella del consumatore sono andate mutando.

Dal lato dell'imprenditore, l'elevato tasso di disoccupazione giovanile spinge molti giovani a cercare lavoro autonomo. Partendo da limitate disponibilità di capitale proprio, i "nuovi imprenditori" partecipano a competizioni/bandi internazionali e cercano fondi su internet. Vedi il nuovo fenomeno del crowdfunding.

15 Informazioni estratte dal documentario “Adriano Olivetti” di La Storia Siamo Noi (Rai).

16 Informazioni estratte dal documentario "Aristide Merloni" di La Storia Siamo Noi (Rai). 
Prendendo ispirazione dalla micro-finanza e dal crowdsourcing ${ }^{17}$, il crowdfunding permette ad aspiranti imprenditori di ottenere finanziamenti in rete, attraverso donazioni da parte di chiunque sia interessato al progetto proposto. L'imprenditore non deve fare altro che descrivere il proprio progetto su una delle piattaforme di crowdfunding, stabilire l'ammontare di finanziamento che intende ottenere e poi aspettare che qualcuno contribuisca, con la possibilità di interagire con gli interessati (per esempio attraverso updates sul prodotto, lo scambio di commenti, il posting di un video che illustri il prodotto ideato, ecc.) e di promettere compensazioni (ad oggi è permesso solo promettere dei rewards materiali in cambio di un determinato contributo, come lo stesso prodotto a cui l'imprenditore intende dar vita una volta conclusa la attività di fundraising e di produzione). Alcune iniziative hanno avuto grande successo in termini di fundraising, tant'è che sempre più accademici stanno studiando questo nuovo fenomeno come potenziale alternativa a fondi di angel investing e venture capital. Uno su tutti è il famoso caso del Pebble watch nel 2012, un orologio che si collega all'iPhone e gli smartphone Android tramite Bluetooth, ed avverte chi lo indossa, con una vibrazione silenziosa, riguardo alle chiamate in arrivo, messaggi di posta elettronica e agli sms. L'iniziativa è stata pubblicizzata su Kickstarter, una piattaforma di crowdfunding americana tra le più importanti al momento, ed è stata realizzata grazie ad una raccolta fondi pari a $\$ 10,266,844$, con investimenti da parte di 68,928 persone.

Gli imprenditori che hanno dato vita a queste piattaforme di crowdfunding, che guadagnano nella maggior parte dei casi una percentuale sul finanziamento totale ottenuto dall'imprenditore, hanno dunque saputo leggere il contesto di grande difficoltà economica, soprattutto nel campo dell'occupazione, e hanno data vita ad un modo innovativo di concedere credito a chi ha il potenziale per avviare un'attività imprenditoriale e la necessità di ridurre il costo per provare a lanciarsi e, così facendo, danno un forte impulso alla ricerca e al raggiungimento dell'innovazione.

Al contempo, la crisi non ha solo contribuito a modificare l'identikit dell'imprenditore, ma anche la mentalità del consumatore.

Si stanno ad esempio sviluppando piattaforme che aiutano a definire forme di consumazione aggregata, la cosidetta sharing economy. Si parla di car-sharing, coworking, di siti come Eatfeastly.com, con cui si può riempire casa di sconosciuti e far pagare a ciascuno una quota della spesa alimentare; Yerdle.com dove è possibile scambiare oggetti che non ci servono più attraverso l'impiego di una nuova moneta di scambio elettronica; Airbnb che fa incontrare chi ha una casa con chi vuole prenotare un alloggio breve. E vi sono anche tanti esempi di start-up italiani che si adoperano in questo campo, come Dropis, sito che permette lo scambio di beni e servizi utilizzando una specifica moneta elettronica, e Skillbros, definito come un $e$ bay delle competenze, ovvero un sito dove è possibile insegnare e/o prendere lezioni da chi ne ha le competenze.

17 Definito come l'esternalizzazione di un determinato compito ad un grande gruppo di persone, in forma di un invito aperto (Howe, 2006). 
In linea con questa crescente interazione online tra gli utenti, i siti di condivisione di informazione sono diventati una costante in espansione del nostro tempo. In Italia, Mutui Online ha dato la possibilità a chi è in cerca di un mutuo di poter comparare le diverse offerte proposte dai diversi player nel mercato; l'imprenditore Dennis Crowley ha fondato Foursquare, il social network che permette di conoscere i posti di maggiore attrattività in ogni città, leggendo i commenti di altre utenti; Pinterest, ha creato un portale affinché le persone possano condividere foto e racconti su qualunque cosa sia di loro interesse.

Sono sempre di più quindi le piattaforme che permettono l'incontro di un numero crescente di utenti, un modello di business che fa dell'informazione il suo punto di forza: le piattaforme guadagnano dalla pubblicità effettuata sul loro sito.

In alcuni casi la figura dell'imprenditore e del consumatore è andata persino fondendosi. “...(I consumatori) sono spesso autori di nuove soluzioni ed esperienze, che diventano concorrenti con le offerte delle imprese: si pensi al fenomeno del 'Couchsurfing ${ }^{18}$, una modalità comunitaria di ospitalità che si è affermata a livello internazionale, inventata interamente dai consumatori... Questo è un grande cambiamento economico e sociologico, amplificato da internet e dalla conseguente rivoluzione dei modi di comunicazione e di interazione tra gli individui..." dice Antonella Carù, ordinario di Economia e Gestione delle Imprese presso il Dipartimento di marketing della Bocconi, in un'intervista del 10/02/2011 http://www.viasarfatti25.unibocconi.it/notizia.php?idArt=7138 ${ }^{19}$.

\section{Conclusioni}

L'innovazione è una variabile fondamentale per la crescita di un Paese. Dare vita a nuove tecnologie, nuovi servizi, nuovi formule produttive, genera valore non solo per le imprese coinvolte nel processo innovativo e, attraendo talenti e creando spazi per gli individui più creativi, contribuisce a creare un modello di crescita inclusiva e sostenibile per l'intera comunità.

Questo racconto ha avuto dunque come obiettivo quello di portare alla luce alcuni degli episodi di maggior successo che testimoniano quanto detto sopra. Dall'innovazione di prodotto all'innovazione come nuovi modelli di business, dagli investimenti delle imprese nel sociale a figure di imprenditori che hanno rivoluzionato il modo di interagire con i propri lavoratori e con il proprio territorio.

Questo perché è fondamentale continuare a sostenere l'innovazione e dunque gli imprenditori, il veicolo tramite cui l'innovazione può essere tradotta in benefici economici, tecnici e sociali per la comunità.

18 Un servizio di ospitalità con il quale una persona mette a disposizione il proprio divano di casa per un viaggiatore, offrendo in pratica un'alternativa agli ostelli, hotel e alberghi.

19 I consumatori nuovi protagonisti dell'innovazione. Come sfruttare - eventualmente dopo averle create - le competenze di chi compra". Intervista apparsa su Sarfatti 25, il 10/02/2011. Riferimento al quarto sito web citato nella bibliografia. 
Ed è particolarmente importante continuare a farlo in Italia.

Come sottolineato nel report di Idee per la Crescita del 23 Aprile 2013 "Proprio perché è un Paese ricco, l'Italia ha già sfruttato le opportunità di crescita offerte dalle sue tecnologie e dalla sua organizzazione sociale del passato. Non può più crescere per imitazione e l'enorme accumulo di debito pubblico del passato vieta anche di crescere con la spesa pubblica che anzi dovrà diminuire per fare spazio a una riduzione del carico fiscale su famiglie e imprese. Per l'Italia di oggi non c'è alternativa alla crescita "per innovazione", che passa attraverso la produzione di idee e la loro trasformazione in imprese" (Università Bocconi - EIEF 2013, p. 1).

\section{Bibliografia}

AAKER D. (2011), Brand Relevance. Marketing innovative per spiazzare i competitori. FrancoAngeli, Milano.

BADEN-FULLER C., MACMILLAN I. C., DEMIL B., LECOCQ’S X. (2010), “Business models as models (editorial)", Long Range Planning, vol. 43, n. 2-3, pp. 143-145.

CHRISTENSEN M. C. (1993), "The rigid disk drive industry: a history of commercial and technological turbulence", Business history review, n. 67 (winter 1993), pp. 531-588.

CLARK B., HENDERSON M. (1990), "Architectural innovation: the reconfiguration of existing product technologies and the failure of established firms", Administrative Science Quarterly, vol. 35, n.1, Special Issue: Technology, Organizations, and Innovation, pp. 9-30.

FOSFURI A., GIARRATANA M.S., ESTHER R. (2010), "Community-based strategies in action: building and sustaining a product differentiation advantage", Open Access publications from Universidad Carlos III de Madrid info:hdl:10016/9311, Universidad Carlos III de Madrid.

GARDINER J.P. (1986), "Design trajectories for airplanes and automobiles during the past fifty years", Christopher Freeman (ed.), Design, Innovation and Long Cycles in Economic Development, Francis Printer, London, pp. 121-141.

GAMBARDELLA A., MCGAHAN A.M. (2010), "Business-model innovation: general purpose technologies and their implications for industry structure", Long range planning, n. 43, pp. 262-271.

HENDERSON R.B., CLARK K. B. (1990), "Architectural innovation: the reconfiguration of existing product technologies and the failure of established firms", Administrative Science Quarterly, vol. 35, n.1, Special Issue: Technology, Organizations, and Innovation, pp. 9-30.

HOWE J. (2006), “The Rise of Crowdsourcing”, Wired Magazine, Issue 14.06.

MILLER R., SAWYERS D. (1968), The technical development of modern aviation, Praeger, New York.

NESTA (2012), "Plan I: The Case For Innovation-led Growth", Nesta website http://www.nesta.org.uk/publications/plan-i

SCHUMPETER J.A. (1911), The Theory of Economic Development: An inquiry into profits, capital, credit, interest and the business cycle, Harvard Economic Studies 46.

SCHUMPETER J.A. (1941), An economic interpretation of our time: The Lowell Lectures. The Economics and Sociology of Capitalism, Princeton University Press, Princeton, N.J., pp. 339-400. 
SCHUMPETER J.A. (1942), Capitalism, Socialism and Democracy, Routledge, Taylor \& Francis Group, New York.

TORRISI S. (2004), "Innovazione tecnologica, competenze e strategie competitive delle imprese. Il caso del software", Sinergie n. 64-65/04, pp. 301-329.

YUNUS M., MOINGEON B., LEHMANN-ORTEGA L. (2010), "Building social business models: lessons from the Grameen experience", Long Range Planning, n. 43, pp. 308325 .

\section{Sitografia}

http://osep.northwestern.edu/projects/ilab

http://scofieldglobal.wordpress.com

http://www.life-global.org/en/LEARN-ONLINE/HP-Life-e-Learning

http://www.viasarfatti25.unibocconi.it/notizia.php?idArt=7138

http://www.nesta.org.uk/publications/plan-i 